\title{
Realismo renovado y Amor y guerra (1999) de Ramón Saizarbitoria
}

\author{
MARI José OlAZIREGI
}

El objetivo de este breve artículo será analizar las ideas que sobre el Realismo propuso el escritor francés Alain Robbe-Grillet y la influencia que éstas pudieron tener en la configuración de la novela Bihotz bi (1996) (Amor y Guerra, Espasa-Calpe, 1999, trad.: Bego Montorio), del escritor vasco Ramón Saizarbitoria. Trataremos de reflexionar en torno a la novedad y especificidad del nuevo enfoque que se propone desde los escritos y novelas del escritor francés Robbe-Grillet. En este sentido, su oposición clara a la novela balzaciana, sus clara influencia de la Fenomenología de Husserl y Merleau Ponty, la cada vez más manifiesta concepción estructuralista que subyace a su obra...serán algunos de los puntos que servirán de guía para nuestro análisis. En una segunda fase, y tras contextualizar muy brevemente la actual narrativa en lengua vasca, disertaremos sobre el lugar canónico que en él ocupa el escritor Ramón Saizarbitoria y analizaremos una de sus novelas, la arriba mencionada Bihotz bi. A través de los ejemplos que iremos extrayendo de la misma, realizaremos una lectura comparatista de las estrategias narrativas que el autor ha utilizado, incidiendo, sobre todo, en la idea de realismo que subraya el texto.

\section{REALISMOS}

Son pocos los conceptos que, en el ámbito de la investigación literaria, han resultado tan polisémicos, controvertidos y debatidos como el concepto de Realismo. De hecho, sería más acertado hablar de Realismos y, en este sentido, hacemos nuestra la afirmación de R. Williams cuando dice que «Yet realism is not an object, to be identified, pinned down, and appropiated. It is, rather, a way of describing, quite naturally, have varied, in the ordinary exchange and development of experience» (Williams, R., 2001: 300). Vemos que, por un lado, la conceptualización del Realismo se ha asociado al problema de la representación, es decir, a la idea de que los textos literarios «representan» algo (mundos físicos, psíquicos...). Esta idea que implica la distinción entre el texto y el mundo, está implícita en nociones como la de mímesis o imitación, la de realismo y naturalismo...o en las recurrentes metáforas que los escritores han utilizado a lo largo de los siglos (como, por ejemplo, la conocida definición de Stendhal cuando 
afirmó que la novela era un espejo que se paseaba por la calle). En definitiva, preguntas básicas como: ¿Hace la literatura referencia a algo externo a los textos? ¿Qué tipo de «verdad» busca el hecho literario?, son las que subyacen a las teorías que, hasta la irrupción de los postestructuralistas, han tratado de explicar la naturaleza de la «representación» literaria. Y decimos que el Postestructuralismo (incluidos el New Historicism, el Feminismo, o la Deconstrucción), subvirtió la dicotomía mundo-texto, porque tras las afirmaciones de M. Foucault o J. Derrida, toda distinción entre literatura y mundo resultaba impertinente. ${ }^{1}$ En cualquier caso, es evidente que las diferentes teorías en torno a lo que la literatura representa, han tratado, desde planteamientos epistemológicos diversos, responder a la pregunta de qué aspecto de la vida es el que los textos literarios representan: si tratan de dar una imagen exacta o simbólica del mundo exterior, si revelan las características universales de la naturaleza humana, o si, en cambio, su objetivo es mostrar las formas ideales que subyacen a los objetos del mundo exterior. Son aproximaciones que, en definitiva, podrían ser agrupadas en dos grupos importantes; por un lado, aquellas que, cercanas al idealismo filosófico platónico, consideran al mundo exterior «as a world of mere appearances, of dead matter, to be brought to life only by the mythopoetic power or the philosopher poet $»{ }^{2}$ por otro, aquellas que partiendo de la importante teoría de la representación de la Poética de Aristóteles, incorporan y reinterpretan el concepto de Mímesis. Entre éstas últimas, podríamos mencionar, entre otros, a Emile Zola y el Naturalismo que, gracias a la aplicación del método experimental de las teorías médicas de C. Bernard, proponía que la novela debía basarse en la observación y en la experimentación para establecer las causas psicológicas de las relaciones sociales. Como afirma R. Selden (1997: 42), Zola contradice a Aristóteles cuando afirma que el novelista representa más lo concreto y lo individual que lo abstracto y lo general. Por otro lado, también podríamos recordar las reinterpretaciones que desde la Crítica Marxista se han hecho del concepto de Realismo, y subrayar los debates que en su seno de han desarrollado desde planteamientos contrapuestos (p.e. el debate entre B. Brecht y G. Lukácks). Para Lukácks, los novelistas realistas del siglo XIX ${ }^{3}$ habrían realizado en sus novelas,

1 Foucault, M: 1970, The order of Things: An Archeology of the Human Sciences, Random House, NY, p. 290: «if language expresses, it does so not in so far as it is an imitation and duplication of things, but in so far as it manifests...the fundamental will of those who speak it'. Derrida, J., 1976, Of Grammatology, The John Hopkins University Press, Baltimore, p. 163: «There is nothing outside the Text».

SELDEN, S. (1988) 1997: op. cit., p. 9.

${ }^{3}$ Es importante señalar la importancia que la novela realista del siglo XIX ha tenido para los teóricos que han tratado de reflexionar sobre la cuestión del realismo en la investigación literaria. Por mencionar sólo dos ejemplos, ahí tenemos, además del mencionado G. Lukácks, a Raymond Williams, quien en su conocida The Long Revolution (Broadview Press, Toronto, 2001. originariamente publicado en 1961), tras afirmar que la novela realista «pasó de moda junto con los coches de caballos» (pág.. 303), continúa subrayando las supuestas ventajas que aquel realismo tenía en oposición al realismo imperante en las novelas modernas: «Yet realism as an intention, in the description of these states, has not been widely abandoned. Is it merely that 'everyday, ordinary reality' is now differently conceived, and that new tecnhiques have been developed to describe this new kind of reality, but still with wholly realistic intentions? The questions are obviously very difficult, but one way of approaching an answer to them may be to take this ordinary belief that we have 
junto a la representación de la realidad, un análisis crítico de las relaciones y tensiones de los distintos grupos y clases de la sociedad, apuntando posibles vías de evolución de la misma. En este sentido, Lukácks concebía la tarea creadora del escritor como una contribución a la reforma de la sociedad futura, como una forma de «praxis social». El desacuerdo de B. Brecht con Lukácks vino, precisamente, en la cuestión de la forma, pues, tras afirmar que la realidad es cambiante y que por ello las formas de representación también deben serlo, Brecht defendió que la novela no tiene por qué ser considerada como la forma ideal para reflejarla y que técnicas como las utilizadas por los modernistas, calificados de decadentes por Lúkacks, podían ser usadas por los socialistas para reflejar la realidad. ${ }^{4}$ Erich Auerbach, por su parte, en su conocido Mímesis: La realidad en la literatura (FCE, México, 1950) planteó una aproximación innovadora al concepto de realismo, al relacionarlo con la evolución de las formas literarias. ${ }^{5}$

Sin duda alguna, si algún mérito es atribuible al Nouveau Roman es el hecho de haber dado pie a toda una «ère du soupçon» con respecto a elementos que para la novela realista tradicional eran absolutamente imprescindibles, como pueden serlo la trama y su psicología. Para estos escritores, el arte busca plantear preguntas, ${ }^{6}$ y para ello, es la investigación formal, la experimentación, la única vía que el artista tiene a su mano. Vistos desde hoy, estos escritores que siempre negaron constituir un grupo, ${ }^{7}$ o que incluso fueron calificados provocativamente por uno de sus miembros, Nathalie Sarraute, como «une association de

abandoned (developed beyond) the realistic novel, and to set beside it my own felling that there is a formal gap in modern fiction, which makes it incapable of expressing one kind of experience, a kind of experience which I find particularly important and for which, in my mind, the word 'realism' keeps suggesting itself» (op. cit. Páginas 303-4). Para Williams, el realismo clásico acertó en crear $y$ juzgar «the quality of a whole way of life in terms of the qualities of persons» (p. 404). Eso es, precisamente. lo que le pide a la novela, que trate de reflejar la vida de los seres humanos, pero no como seres aislados, sino como miembros de un modo de vida.

+ Selden, S. (1988) 1997: op cit., pp. 42-43. Cf. LuKÁCKS, G., «Art and Objective Truth», in Writer and Critic and Other Essays, Merlin Press, London, 1970; Brecht, B., «Alienation Effects in Chinese Acting» and «The Popular and the Realistic», in Brecht on Theatre, Hill \& Wang, NY, 1964.

5 SELDEN, S. (1988) 1997: op cit., pp. 43: «by linking it with stylistic development over long historical periods. He argues that in the classical period the 'separation of styles' (...) prevented the development of realism in the modern sense, because only characters of the highest social standing could be trated as tryly serious. (...) Only in the modern novel does the writer present mundane reality in its historical particularity and with full seriousness».

${ }^{6}$ RobBe-Grillet, A., (1963:14): «Car la fonction de l'art n'est jamais d'illustrer une vérité -ou même une interrogation-connue à l'avance, mais de mettre au monde des interrogations...».

Butor, M. (1975:83): «Historiquement, l'expression «nouveau roman» a déjà un sens assez clair: il s'agit d'un certain nombre de romanciers qui sont devenus brusquement plus connus vers 1956. Ces romanciers, fort divers, avaient évidemment des points communs, et ce n'est pas un hasard s'ils on été publiés alors en grande partie par la même maison d'édition. Dans les cours que j'ai donnés sur le roman français aux XXe siècle, j'ai été bien obligé de présenter les choses de cette façon, et d'admettre qu'à cet égard, je fais partie du «nouveau roman». Mais un tel rapprochement n'a nullement permis la constitution d'une doctrine commune, et j'ai été longtemps agacé de voir des critiques, sous prétexte de «nouveau roman», m'attribuer des «théories» qui n'étaient nullement les miennes, ce qui multipliait les malentendus.». I hid. RobBE-Grillet, A. (1963:9). 
mal faiteurs», revolucionaron el concepto de representación en los años en que se desarrolló su actividad (entre 1954 y 1959, según R. M. Albérès (1974)). En cualquier caso, su aportación ha sido reconocida, no sólo por los escritores «estructuralistas» que en los 60 se agruparán en torno a la revista Tel Quel (Sollers, Faye, Cayrol, Pleynet, Thibaudeau y J. Ricardou), sino por la institución literaria que no dudó en otorgar el Premio Nobel a uno de sus integrantes, C. Simon, en 1985. El afán por la renovación con el que irrumpió en el panorama de los años 50, hace que el Nouveau Roman pueda ser incluido en la tradición modernista (A. E. Babcock 1997:133). ${ }^{8}$ Como se sabe, la obsesión modernista por la técnica narrativa fue muy influyente en aspectos como el del punto de vista, o focalización. La defensa que, por ejemplo, Henry James realizara en sus conocidas «Critical Prefaces» sobre la adecuación de que el narrador adquiriera un punto de observación y abandonara la narración omnisciente decimonónica, influyó de modo importante no sólo en toda la narrativa de la primera mitad del siglo Xx, sino en trabajos críticos clásicos como el de P. Lubbock (cf. The Craft of Fiction, 1921), y otros posteriores. Este relativismo imperante en los diferentes ámbitos del pensamiento y del arte de principios de siglo, derivó en una aproximación a la Fenomenología en los escritores del Nouveau Roman. En efecto, novelas como Le voyeur (1955) o La Jalousie (1956) incorporaban el modo de narrar behaviorista o punto de vista objetivo, pero la novela ya no pretendía representar un mundo observable, sino que lo creaba. ${ }^{9}$ Este «nuevo realismo» (sic) que Alain Robbe-Grillet explicó en su conocido Pour un nouveau roman (1963), en lugar de un universo cargado de significados (psicológicos, sociales, funcionales), pretende crear un mundo más inmediato, un mundo en el que los objetos se impongan por su presencia. ${ }^{10}$ Para ello, en «Une voie pour le roman futur», el escritor francés defendió la necesidad de optar por un lenguaje literario que controlara el uso de palabras con valor analógico o mágico, y optara por adjetivos ópticos y descriptivos (Robbe-Grilllet, 1963: 27). La nueva novela, según

${ }^{8}$ BAвсOCK, A. E. (1997:133): «The error of those who are opposed to the New Novel, in Robbe-Grillet's opinion, isto elevate Balzac's novelistic practice to the status of an absolute standard for fiction writing when in fact it succeeded only because it was suited, in relativistic fashion, to the ideology of a particular time and place. It is significant that most of Robbe-Grillet's arguments are directed against the realist novel of the first half of the nineteenth century and certain of its techniques; the modernist novelists were rebelling agains the same conception of the novel, and in many respects Robbe-Grillet's «revolution» sounds like a continuation of the modernists".»

${ }^{4}$ Deduck, P. A. (1982: 9 and 14): «Reality, then, in the modern novel, is no longer to be recorded or reflected; rather, it is to be created (...). Most of the new experimental novelists would vehemently deny that their work is anti-life or even anti-art, and like Butor, for example, would defend experimentation precisely because it imitates human life in process, and in so doing, affirms it. (...) Indeed, it is Butor and some of his disciples who prefer to be called «neo-realist», rather than "anti-novelist", and who use the term «neo-realism», rather than «anti-roman», in reference to the new novel.»

${ }^{10}$ BARThes, R. (1964:30-31): «L'écriture de Robbe-Grillet est sans alibi, sans épaisseur et sans profondeur: elle reste à la surface de l'objet et la parcourt également sans privilégier telle o telle de ses qualités: c'est donc le contraire même d'une écriture poétique. (...)La condition de l'homme c'est d'être là». Robbe-Grillet rappelait ce mot de Heidegger à propos de En attendant Godot. En bien, les objets de Robbe Grillet, eux aussi, sont faits pour être là. Tout l'art de l'auteur, c'est de donner à l'objet un «être là» et de lui ôter un «être quelque chose»». 
Robbe-Grillet, debía desterrar usos que perduraban desde la novela realista del XIX. Es esto lo que, precisamente, el autor trata de explicar en «Sur quelques notions périmées» (1957). En él afirma que la novela de personajes pertenece al pasado, a una época que marca el apogeo del individuo y donde el ser humano creía comprender y controlar el mundo (1963:63). Pero además, Robbe-Grillet cuestionaba la necesidad de que una novela tuviera que contar una historia («Raconter est devenu proprement impossible», Robbe-Grillet, A., 1963:37), o que el escritor tuviera que estar comprometido, y volvía a incidir en la imposibilidad de distinguir entre el fondo y la forma de una obra literaria (R. G., 1963: 50). " En definitiva, tal y como trató de argumentar en "Nature, humanisme, tragédie» (1958), Robbe-Grillet defendía que la novela debía limitarse a la descripción externa de las cosas, presentarlas como cosas carentes de significado, puras presencias (1963: 131). Para conseguirlo, la vista se presentaba como el sentido privilegiado (de ahí la denominación de «École du regard» al Nouveau Roman), y la descripción óptica como el medio adecuado. Y es que ya lo dijo M. Merleau-Ponty: «L'être object n' est donc pas un être-pour-le-sujetpensant, mais un être pour le regard.» ${ }^{12}$ Esa mirada le sirve al sujeto para definir su situación en el mundo (1963: 82), y, en definitiva, es en esa relación con los objetos que le rodean, en ese subjetivismo extremo, donde el sujeto se define. Es esta subjetividad total la que, en definitiva, la nueva novela francesa se proponía (1963: 148).

La novela se convierte, de este modo, en una obra que invita a participar en su creación (Robbe-Grillet, 1963: 169), un texto escriptible, que diría R. Barthes (cf. $S / Z$ ). Y esa creación nos recuerda que las realidades que la novela construye son realidades de ficción, de lenguaje (Robbe-Grillet, A.1963: 175). Como vemos, Robbe-Grillet subvierte el concepto tradicional de realismo, entendido como mímesis o representación de una realidad, y hace suya la afirmación estructuralista de que «What takes place' in a narrative is from the referential (reality) point of view literally 'nothing'; 'what happens' is language alone, the adventure of language, the unceasing celebrarion of its coming». ${ }^{13}$ Esta aproximación a planteamientos estructuralistas hace que la que se ha denominado «Nouveau Nouveau Roman» (cf. Babock, A.E., 1997), es decir, las obras que se publican en la década de los 60 y 70 , estén mucho más conectadas al posmodernismo que las obras de los años 50. En este sentido, es más que reseñable que los escritores del Nouveau Roman que han seguido publicando en décadas posteriores, hayan abandonado la experimentación extrema de los primeros tex-

$"$ Ibid. Beckett, S. (1983) «Dante... Bruno. Vico...Joyce», in Disjecta, John Calder, London, p. 27: «Here form is content, content is form. You complain that this stuff is not written in English. It is not written at all. It is not to be read -or rather it is not only to be read. It is to be looked and listened to. His writing is not about something; it is that something itself. (...) When the sense is sleep, the words go to sleep.(...) When the sense is dancing, the words dance. (...) The very words are tilted and effervescent. (...) Here is the savage economy of hieroglyphics. Here words are not the polite contortions of $20^{\text {th }}$ century printer's ink. They are alive. They elbow they way on to the page, and glow and blaze an fade and disappear.

12 Merleau-Ponty, M.: 1945, Phénomenologie de la perception, Gallimard, París, p. 292.

13 Barthes, R., 1977: «Introduction to the Structural Analysis of Narratives», Image-MusicText, Fontana, London, 124. 
tos y se hayan acercado, como en el caso de Robbe-Grillet o C. Simon, a universos novelescos donde la autobiografía y la memoria histórica sirven de excusa literaria para crear un universo narrativo. ${ }^{14}$ En cualquier caso, aunque la adscripción del Nouveau Roman sea debatida en la historiografía literaria del siglo XX (se ha disertado mucho en torno a su adscripción al modernismo o al posmodernismo, así como al existencialismo o al estructuralismo), es reseñable que seguramente se trate del último movimiento literario. ${ }^{15}$ Un movimiento que, como hemos visto, renovó el concepto de realismo.

\section{NARRATIVA VASCA CONTEMPORÁNEA: LA OBRA DE RAMÓN SAIZARBITORIA}

Tal y como hemos querido reflejar en anteriores publicaciones, ${ }^{16}$ la trayectoria novelesca de Ramón Saizarbitoria (Donostia, 1944) marcó un hito definitivo en la modernización de la novela vasca. Pero además, fue su poética experimental la que definió el canon literario de la novela vasca en la década de los 70 y nos permitió sintonizar con las corrientes literarias europeas de la época.

Saizarbitoria hace suya la afirmación de J. Ricardou de que la novela es la aventura de una escritura. ${ }^{17}$ Esa aventura, en el caso de la novelas del autor vasco, hacía suyas las técnicas narrativas de los textos clásicos del Nouveau Roman, en especial el tono frío de la narración behaviorista, para luego pasar, en las novelas de la década de los 90, a universos literarios que toman por eje la memoria. ${ }^{18}$ En total, Saizarbitoria ha publicado cuatro novelas, un libro de narraciones, una novella para jóvenes y un libro de ensayos. Al hilo de lo planteado por $\mathrm{M}$. Butor, su novela se convirtió, en la década de los años 70, en centro de experimentación formal, centro que trataba de sugerir realidades nuevas con formas narrativas nuevas. Su primera novela Egunero hasten delako [Porque comienza cada día] (1969) narra, en dos planos narrativos, la historia de una joven estu-

${ }^{14}$ Cf. Flieder, L., 1998: Le roman français contemporain, Seuil, París, chapter 7.

15 Cf. BABCOCK, A. E. (1997: 140).

16 Cf. Olaziregi, M. J.: «Encuentros y desencuentros con la literatura vasca», Revista de Lenguas y Literaturas catalana, gallega y vasca VII, 379-387, 2002.

17 «(...) Jean Ricardou a défini le roman moderne comme étant non plus «l'écriture d'une aventure, mais, au contraire, l'aventure d'une écriture», et, de son côté, Olga Bernal a très justement souligné que «si le roman du XIXe siècle était un roman du savoir, le roman moderne est essentiellement un roman du non-savoir.» (AA.VV. (1972), Nouveau Roman: hier, aujourd' hui. 2. Pratiques, Union Générale d'Editions. Col. 10/18, París, 84).

18 Dentro de los «Encuentros de Narrativa Castellana, Gallega, Catalana y Vasca» organizados por la Universidad Nacional de Educación a Distancia española (Málaga, 14-3-2001), Saizarbitoria afirmaba: "La recuperación de la memoria está muy presente en mi aventura narrativa. Si uno no tiene problemas de memoria, si recuerda con pelos y señales hasta el último detalle la historia que pretende narrar, si no tiene ninguna duda, ninguna sombra, difícilmente, desde mi perspectiva, tendría nadie interés en contarla a no ser que pensase que puede seducir al oyente o por puro desahogo. (...) Uno no sabe por qué, en determinado momento, algo que ve, que recuerda o que oye, le impulsa a saber más, a conocer la historia de la que forma parte y, en ese empeño, escarba en su imaginación, en su memoria, tratando de recomponer un todo coherente a partir de unos datos fragmentarios». 
diante que quiere abortar. La referencia que se hace en la novela a la revista Les Temps Modernes o el nombre de la protagonista, Gisèle, que nos recuerda a la famosa abogada que participó en el juicio de Bobigny en 1972, tratan de contextualizar la historia principal. Técnicamente, subrayaríamos el estilo behaviorista con el que se narra el plano principal, los continuos cambios de focalización, o las transcripciones de diálogos al estilo de La chute (1956). También en su segunda novela, Cien metros (Ed. Nueva Cultura), el narrador recurre a diferentes planos narrativos, aunque en esta novela era más evidente la utilización de técnicas fílmicas próximas a la Nouvelle Vague. En cualquier caso, el hecho de que la historia principal que se narraba girara en torno al relato de los últimos cien metros de un activista de ETA antes de ser abatido por la policía, condicionó poderosamente las lecturas erróneas que en su día se hicieron de la novela. Su tercera novela, Ene Jesus [iAy Dios mio!] (1976), obtuvo el Premio de la Crítica en 1976, y podría ser descrita como una metanovela. La imposibilidad de poder contar, vivida de modo trágico por el protagonista, se plasma en un texto fragmentario, donde los elementos narrativos se van simplificando al máximo. Personajes como Samuel y el mudo, junto con las características mencionadas, nos señalan el intertexto más inmediato de la novela: Malone meurt (1951), del escritor irlandés Samuel Beckett. Tras un período de silencio de 19 años, Saizarbitoria volvió a irrumpir en el panorama literario vasco con una novela, $\mathrm{Ha}$ maika pauso (Los pasos incontables, Espasa Calpe, 1998), que volvió a obtener el Premio de la Crítica. Se trataba de las primera novela del autor que tomaba como eje narrativo la memoria, y en este sentido, podríamos considerarla una novela testimonial. Las afirmaciones que el narrador pone en boca de Claude $\mathrm{Si}$ mon («la memoria es como un plato roto»), nos recuerdan que la «restitución» del pasado es una excusa para profundizar en la memoria de toda una generación que vivió la represión franquista de los años 60-70. En definitiva, es el horror y la terrible soledad ante la muerte la que resume el entramado temático de la novela, presentando, para ello, toda una serie de referencias intertextuales y metanarrativas y trayendo a colación citas de escritores, sociólogos, filósofos o músicos. Los pasos incontables es un gran palimpsesto que, gracias a una factura compleja y atractiva, nos transmite una intensidad narrativa excepcional. Con su último libro, Gorde nazazu lurpean (2000) (Guárdame bajo tierra, Alfaguara, 2001), Saizarbitoria volvió a convencer a público y crítica, y el libro fue galardonado con el Premio Euskadi-2000 y el Premio de la Crítica-2000. Es el motivo de la exhumación de cadáveres el que sirve de nexo temático para las cinco historias que integran el libro. Se trata, en realidad, de narraciones basadas en tres exhumaciones reales: las realizadas por el pintor prerrafaelita Dante Gabriel Rossetti para recuperar los poemas que enterró junto a su mujer; la exhumación del cadáver de Yves Montand para realizar una prueba de paternidad, o la exhumación del cadáver de Sabino Arana, el padre del nacionalismo vasco, para trasladarlo de lugar. Junto a éstas, tendríamos otras exhumaciones ficticias que sirven de excusa literaria para las otras dos narraciones. Pero lo que en realidad exhuma el autor son sus propias obsesiones, obsesiones que giran en torno a las terribles vivencias de los gudaris vascos en la Guerra Civil, o a la incomunicación entre hombres y mujeres. Tal y como resumió A. Gabantxo en el The Times Literary Supplement (n. 5249, 7-11-2003, 24): «Through the symbolism of burial, Saizarbitoria suceeds in presenting many defining aspects of the Basque co- 
llective psyche, including obsesión with the past and emotional paralysis. All five novellas are questioning, open-ended; they vibrate with a powerful misture of anger, sarcasm and compassion».

Amor y Guerra (Espasa Calpe, 1999; orig.: Bihotz bi. Guerrako kronikak, Erein,1996) obtuvo el Premio Difusión del Gobierno Vasco en 1996. La novela narra la historia de la degradación de las relaciones conyugales de una pareja, la formada por el narrador - cuyo nombre no es citado- y su mujer, Flora. Al comienzo de la novela, el narrador confiesa que ha matado a su mujer, arrojándola por la ventana de la cocina del piso en el que habitan. El hilo narrativo establece a partir de ese punto un salto atrás, una analepsis, a través de la cual el narrador-protagonista relata los pasos que siguió para planificar el asesinato y trata de recordar los episodios más importantes de la «guerra» doméstica de la pareja. Los sucesivos adulterios de la pareja, las continuas persecuciones y vigilancia a la que la esposa es sometida por parte del marido... son sólo algunos de los hechos que se narran. Junto a éstos, cabría mencionar la otra guerra a la que se le hace referencia en la novela, la Guerra Civil española, que es relatada durante los encuentros que la pareja protagonista tiene con un grupo de gudaris ancianos en una sidrería.

Un argumento que a ojos de cualquier lector resulta más que impactante, pero que además está planteando parodiando el estilo de las narraciones policíacas. Si la comparamos con la de Les Gommes (1953), la parodia resulta más irónica pues las artimañas que idea el narrador de Amor y guerra para que el amante de la mujer cargue con la culpa se vuelven en su contra. Además de lo dicho, el hecho de que la novela cuente desde el primer párrafo el final de la misma, ${ }^{19}$ nos sitúa ante un texto donde no se invita al lector a desentrañar ninguna trama, sino que es invitado a colaborar en la aventura de la escritura. Podríamos describir la novela como una confesión, una confesión en la que el narrador busca la complicidad del lector en el relato de los hechos delictivos, complicidad que viene a ser subrayada por las referencias textuales dirigidas al narratario: 'No se si se entiende la diferencia...Por ejemplo...' (p. 89), 'es un pretil ancho, digo esto para quien no lo conozca' (p. 113)...

Para que se produzca una confesión, tal y como sugería M. Bajtin, es necesario que se haya dado una trasgresión de las normas sociales y, en el caso de esta novela, son los mandatos más elementales de la Biblia («no matarás» y «no cometerás adulterio») los que se trasgreden. El cobarde marido, en vez de aceptar el deterioro de la relación y buscar una salida «digna» a la situación (ejemplificada en la novela por el personaje de Beltrán o a través de las canciones de Léo Ferré), prefiere vengarse y matar a su mujer, dando de este modo fin a una historia de amor con el sacrificio del objeto deseado, como diría Bataille. La novela narra, por boca del marido, los momentos fundamentales del deterioro de la relación con su esposa. El tiempo cronológico viene a ser ralentizado y subvertido por las numerosísimas repeticiones y prolepsis que nos sumergen en el

19 «Mi mujer se llamaba Flora. Decidí matarla el día en que, recogiéndose el camisón en la cintura, se me sentó a horcajadas y me pellizcó las tetillas con la demasiado evidente intención de complacerme» (Saizarbitoria, R., Amor y Guerra, Espasa Calpe, 1999, p. 7.) 
tiempo psicológico del protagonista principal. Como defenderá el narrador, la narración de la historia es mucho más atractiva que la historia misma ${ }^{20} \mathrm{y}$, por esta razón, se repiten una y otra vez, detallada y obsesivamente, las mismas escenas conflictivas. Estas repeticiones que, en realidad, no añaden nuevas informaciones al lector, subrayan el proceso de escritura, es decir, el proceso de construcción del Texto, ${ }^{21}$ que en este caso está estrechamente condicionado por el proceso de recordar.

Como el protagonista de una de las novelas anteriores de Saizarbitoria, Ene Jesus (1976), el narrador de Amor y guerra cree que narrar y contar repetidamente la misma historia nos hace más libres («Por decirlo de alguna manera, contra y escuchar historias nos hace más libres', p. 86). No obstante, si en las novelas anteriores de Saizarbitoria era la reflexión metanarrativa la que interrumpía reiteradamente la narración, en Amor y guerra, es el continuo intento de recordar, la activación de la memoria, la que sirve para poner en marcha la narración. Esa memoria toma sustento en los pequeños detalles que se van añadiendo a las escenas que se repiten, detalles que, en realidad, vienen a reforzar la idea de que recordar es siempre imaginar. Esta afirmación que ya fue planteada por Robbe-Grillet en Pour un nouveau roman (1963: 176), ha sido reiterada en las diferentes entrevistas que el autor francés ha concedido ${ }^{22}$ o en su autobiografía Le miror qui revient (Minuit, 1984). Para que esa memoria se active, hay una fecha que actúa como un auténtico corps conducteur en la novela Amor y guerra. Nos referimos a la fecha del 13 de septiembre que marca no sólo la entrada de las fuerzas franquistas a San Sebastián, sino el día en que la pareja de protagonistas se enfada, o el día en que el protagonista conoce a su amante Violeta, etc.

Junto a lo mencionado, sería reseñable el objetivismo del que de nuevo hace gala Saizarbitoria. Los gestos y movimientos de los personajes, la confección de diferentes recetas culinarias... son narradas con detallismo casi flaubertiano. Este narrador que al igual que el protagonista de Le voyeur es vendedor ambulante, nos explica con un detenimiento un tanto sorprendente cómo hay que freír unos huevos (p. 34), cuál es la forma más apropiada para preparar la ensalada o una lasagna (p. 181), cuál es el modo de preparar una merluza (p. 63)....Podría decirse que, contradiciendo lo que G. Flaubert afirmaba sobre los vascos en su conocido Dictionnaire des Idées Reçues, en Amor y guerra se ironiza en torno a la obsesión que el narrador tiene por la comida , es decir, a la excesiva oralidad con la que sublima el deseo. La ironía con que está perfilado este protagonista masculino, no deja lugar a dudas sobre la distancia que el autor implícito mantiene sobre él. Se trata de un hombre que ronda los cincuenta años, aficionado al fútbol y boxeo, y admirador de canciones conocidas como Love story. Su discurso refleja todo un cúmulo de tópicos machistas, como la de que las mujeres

20 ... «quizá porque no he madurado del todo, la cuestión es que, muchas veces, me gusta apreciar los detalles y matices de diferentes versiones de la misma historia; es decir, que prefiero la narración, la trama, a la propia fábula», Amor y Guerra: 20)

21 Utilizo Texto en el sentido barthiano de la palabra. Véase: «From Work to Text», Barthes, R., (1989) The Rustle of Language, University of California Press.

22 The París Review, 1996, Confesiones de escritores. Narradores 2, El Ateneo, Buenos Aires, p. 242: «La memoria pertenece a la imaginación.» 
sucumben a películas del tipo de Tomates verdes fritos o novelones románticos. $\mathrm{Y}$ es que este marido, aunque es capaz de transcribir con detallismo óptico cualquier reacción o movimiento de su mujer, en realidad, no la «ve», sino que la incluye bajo el epígrafe general de «mujer» que, para él, tiene connotaciones negativas. La ceguera de este marido es tal que todas las discusiones que tiene con su mujer a lo largo de la novela, las atribuye a la necesidad de su esposa de autoafirmarse (esposa que, dicho sea de paso, es profesora de piano y más cultivada que él).

A diferencia de Leopold Bloom quien se conformaba con imaginar y repasar con dolorosa reiteración los adulterios de su mujer Molly, el protagonista de la novela de Saizarbitoria no se conforma con ello y persigue a su mujer para tener pruebas de infidelidad. Al igual que a Molly Bloom, a Flora le sobran algunos kilos, y su nombre hace un guiño a la protagonista de la conocida novela del autor irlandés (to bloom $=$ florecer).

A decir verdad, los personajes femeninos principales de la novela, Flora y Mikele de Abando, son víctimas de los hombres. La primera, se siente abandonada por un marido posesivo y celoso (9); la segunda, por el padre del protagonista, quien, tras dejarla embarazada, desapareció del frente. La descripción física o psicológica de ambas mujeres, subraya su fortaleza (13), y resulta especialmente reseñable la constante comparación que se hace de Flora con el cuadro de Hopper La habitación del hotel (1931), cuadro que, sea dicho de paso, sirvió para ilustrar la portada del libro en su primera edición en euskera. La mujer solitaria que aparece sentada en la habitación del hotel sería una buena imagen de esta esposa abandonada que toca, constantemente, una pieza al piano cuya letra no deja lugar a dudas: «Look at me, I am as helpless as a kittem up a tree» (18). En comparación a ella, Mikele de Abando, activista del Partido Nacionalista Vasco durante la Guerra Civil e integrante del Emakume Abertzaleen Batza (103), da una imagen de mujer independiente, ejemplo del supuesto matriarcalismo vasco. Junto a ellas, Violeta es la joven amante que resulta ser sobrina del protagonista y su caracterización está mucho menos perfilada. La tensión en torno a las diferente infidelidades y disputas va in crescendo y varios elementos de la novela funcionan como verdaderos mise en abyme, anticipándonos el trágico final de la esposa del protagonista. Entre ellos, cabría mencionar el grabado de Gustave Doré (164) (Jezabel arrojada por la ventana) que se ve en la página del libro donde decide guardar el mechón de pelo del supuesto amante, o los relatos de los jubilados de la sidrería donde se sugieren escarmientos a la infidelidad (201). Al protagonista le será inútil tratar de volver a «poseer» a su esposa por medio de tórridas escenas de sexo o tratar de demostrarse a sí mismo que todavía "puede», con los escarceos sexuales que tiene con su amante Violeta, 20 años más joven que él. El último escenario de su infidelidad, la cabina de teléfono donde hay expuesto un cartel de «Macho de Guerlain» (132), no deja de ser irónico. Como en muchos relatos detectivescos, la ampliación de una fotografía le revelará al protagonista la identidad de Violeta, pues ésta, al igual que su madre y su abuela, lleva la señal de la muerte en el cuello. Amor, odio, deseo, relaciones matrimoniales que son sentidas como incestuosas (84)...son ingredientes que, sin duda, hacen de Amor y guerra una propuesta atractiva. 
Ramon Saizarbitoria ha sabido mostrar, a través del marido delirante de esta novela, que realismo significa hoy discursividad recurrente. Que todo intento de aproximación realista está abocado a una constante creación y recreación de mundos posibles. Mundos donde el recuerdo se tiñe de imaginación, o donde la descripción supuestamente objetiva queda delatada por esa conciencia fenoménica que no oculta su posición.

\section{BIBLIOGRAFÍA}

AA.VV. (1972): Nouveau Roman: hier, aujourd'hui. I. Problèmes généraux, Ed. 10/18, Paris.

Albérès, R. M. (1974): Littérature. Horizon 2000, Albin Michel, Paris.

BuTOR, M. (1975): Essais sur le roman, Gallimard, Paris.

BaвсOCк, A. E. (1997): The New Novel in France. Theory and Practice of the Nouveau Roman, Twayne Publishers, New York.

BARTHES, R.: Essais critiques, Seuil, Paris.

Deduck, P. A. (1982): Realism, Reality, and the Ficional Theory of Alain Robbe-Grillet and Anais Nin, University Press, Washington.

Fletcher, J. \& Calder, J. (1986): The Nouveau Roman Reader, John Calder, London.

Foucault, M. (1970): The order of Things: An Archeology of the Human Sciences, Random House, NY. Ricardou, J., 1967, Problèmes du nouveau roman, Seuil, Paris.

Olaciregui, M. J. (1999): «Un siglo de novela en euskera», in Urquizu, P. (dir.), Historia de la Literatura Vasca, Uned, Madrid, pp. 504-588.

Robbe-Grillet, A. (1963): Pour un nouveau roman, Gallimard, Paris.

SElden, S. (1988), 1997 (Ed.): The Theory of Criticism. From Plato to the Present, Longman, London.

Stern, J. P. (1973): On Realism, Routledge \& Kegan Paul, London.

Williams, R. (1961), 2001: The Long Revolution, Broadview Press, Toronto. 